УДК 517.555

\author{
A. I. BANDURA
}

\title{
ENTIRE CURVES HAVING BOUNDED $l$-INDEX IN $\ell_{\infty}$
}

\begin{abstract}
A. I. Bandura. Entire curves having bounded l-index in $\ell_{\infty}$, Mat. Stud. 52 (2019), 108-112.
In this paper we propose an approach to introduce a concept of bounded index in an infinite-dimensional space. Our object of investigation is the space $\ell^{\infty}$ equipped with the norm $\|x\|_{\infty}=\sup \left\{\left|x_{n}\right|: n \in \mathbb{N}\right\}$. We consider entire curves from $\mathbb{C}$ to $\ell_{\infty}$ and prove a proposition indicating connection between of the $l$-index boundedness of every component of the curve and the $l$-index boundedness of the curve. Moreover, we obtain sufficient conditions of the $l$-index boundedness of entire curves in the space. They describe local behavior of norm of derivatives of the entire curves on the discs. Also, there is posed a problem on necessary conditions of the $l$-index boundedness of entire curves in infinite-dimensional spaces.
\end{abstract}

1. Introduction. In the last years, analytic functions of several variables having bounded index are intensively investigating. Main objects of investigations are such function classes: entire functions of several variables $[5,6,15,16]$, functions analytic in a polydisc [7], in a ball $[3,4]$ or in the Cartesian product of the complex plane and the unit disc [8].

For entire functions and analytic function in a ball there were proposed two approach to introduce a concept of index boundedness in a multidimensional complex space. They generate so-called functions of bounded $L$-index in a direction and functions of bounded L-index in joint variables. But a starting point for these all investigations is the definition of entire function of bounded index introduced by B. Lepson [14]: a function $f(z), z \in \mathbb{C}$, is called a function of bounded index if there exists a number $N \in \mathbb{Z}_{+}$such that for all $p \in \mathbb{Z}_{+}$ and $z \in \mathbb{C}$ the next inequality

$$
\frac{\left|f^{(p)}(z)\right|}{p !} \leq \max \left\{\frac{\left|f^{(k)}(z)\right|}{k !}: 0 \leq k \leq N\right\}
$$

holds. Later M. Sheremeta and A. Kuzyk $[13,20]$ generalized the concept replacing $p$ ! in the denominator by $p ! l^{p}(|z|)$ (so-called functions of bounded l-index) where $l: \mathbb{C} \rightarrow \mathbb{R}_{+}$is a positive continuous function.

There are papers on analytic curves of bounded $l$-index. This function class naturally appears if we consider systems of differential equations and investigate properties of their analytic solutions. A concept of bounded index for entire curves was introduced with the sup-norm by L. F. Heath [12] and with the Euclidean norm by R. Roy and S. M. Shah [17]. In these papers the authors replaced the modulus of function by an appropriate norm in the definition. Later there were also proposed definitions of bounded $\nu$-index by R. Roy and

2010 Mathematics Subject Classification: 30D20, 30D15.

Keywords: bounded index; bounded $l$-index; entire curve; $\ell_{\infty}$.

doi:10.30970/ms.52.1.108-112

(c) A. I. Bandura, 2019 
S. M. Shah [18] for entire curves with these norms. In these definitions, R. Roy and S. M. Shah replaced $p$ ! by $p !|z|^{p}$ and so on. Also M. T. Bordulyak and M. M. Sheremeta [9,21] studied curves of bounded $l$-index and of bounded $l$ - $M$-index which are analytic in arbitrary bounded domain on a complex plane and $l$ is a positive continuous functions in the domain. They replaced $p$ ! by $p ! l^{p}(|z|)$ in the definition of Lepson and used both mentioned norms. These mathematicians found sufficient conditions providing $l$-index boundedness of every analytic solutions for some system of linear differential equations. A concept of bounded $l$ - $M$-index uses idea of replacement the modulus of derivative by maximum modulus of the derivative, i.e. $\left|f^{(p)}(z)\right|$ by $\max \left\{\left|f^{(p)}(z)\right|:|z|=r\right\}$ in the definition of Lepson.

Moreover, F. Nuray and R. Patterson [16] investigated vector-valued entire functions $F: \mathbb{C}^{2} \rightarrow \mathbb{C}^{2}$ of bounded index. They presented an application to study properties of entire solutions of some system of linear partial differential equations. Also there are papers on properties of vector-valued functions which are analytic in the unit ball and have bounded L-index in joint variables $[1,2]$.

2. Problems and main results. Nowadays all considered spaces in theory of bounded index have finite dimension. The infinite-dimensional spaces were not an object of investigation in the theory of function of bounded index. Prof. A. Zagorodnyuk (2011-2016) repeatedly posed the following question in conversations with the author at the conferences "Modern problems of probability theory and mathematical analysis" (Vorokhta, Ukraine):

Problem 1. Is it possible to construct a theory of bounded index for entire functions $F$ with a countable set of variables, that is, $F: \mathbb{C}^{\infty} \rightarrow \mathbb{C}$ ?

We are not ready to give full answer to the question. But in this investigation we consider some similar problem:

Problem 2 (O. Skaskiv, 2019). Is it possible to construct theory of bounded index for entire curves in an infinite-dimensional complex space, i.e. $f: \mathbb{C} \rightarrow \mathbb{C}^{\infty}$ ?

In these short notices we propose some approach to introduce a concept of bounded index in the space $\ell^{\infty}$ that is the sequence space whose elements are the bounded complex sequences. The space is equipped with the norm $\|x\|_{\infty}=\sup \left\{\left|x_{n}\right|: n \in \mathbb{N}\right\}$.

Let $F: \mathbb{C} \rightarrow \ell^{\infty}$ be an entire curve, i.e. $F=\left(f_{1}, f_{2}, \ldots, f_{n}, \ldots\right)$, where every $f_{j}$ is an entire function, and $\|F(z)\|_{\infty}<+\infty$ for any $z \in \mathbb{C}$.

The notation $F^{(k)}(z)$ stands for $\left(f_{1}^{(k)}(z), \ldots, f_{n}^{(k)}(z), \ldots\right)$.

By $B^{\infty}$ we denote the space consisting of entire curves $F: \mathbb{C} \rightarrow \ell^{\infty}$ which are bounded on every compactum, i.e. $F \in B^{\infty}$ if for every compactum set $G \subset \mathbb{C}$ there exists $C>0$ such that for any $z \in G$ one has $\|F(z)\|_{\infty} \leq C$. The condition provides that any derivative of every function from $B^{\infty}$ also belongs to $B^{\infty}$.

Let $l: \mathbb{C} \rightarrow \mathbb{R}_{+}$be a positive continuous function.

A function $F \in B^{\infty}$ is called a function of bounded l-index, if there exists $m_{0} \in \mathbb{Z}_{+}$such that for every $m \in \mathbb{Z}_{+}$and for all $z \in \mathbb{C}$ one has

$$
\frac{\left\|F^{(m)}(z)\right\|_{\infty}}{m ! l^{m}(z)} \leq \max _{0 \leq k \leq m_{0}} \frac{\left\|F^{(k)}(z)\right\|_{\infty}}{k ! l^{k}(z)} .
$$

The least such integer $m_{0}$ is called the $l$-index of the curve $F$ and is denoted by $N(F ; l)$. If $l \equiv 1$ then $F$ is said to be of bounded index. A simple example of such a curve of bounded index is $\left(e^{z}, e^{z / 2}, \ldots, e^{z / n}, \ldots\right)$. 
Let us denote

$$
\lambda_{\mathbf{b}}(\eta)=\sup _{t_{1}, t_{2} \in \mathbb{C}}\left\{\frac{l\left(t_{1}\right)}{l\left(t_{2}\right)}:\left|t_{1}-t_{2}\right| \leq \frac{\eta}{\min \left\{l\left(t_{1}\right), l\left(t_{2}\right)\right\}}\right\} .
$$

By $Q$ we denote the class of positive continuous function $l: \mathbb{C} \rightarrow \mathbb{R}_{+}$, satisfying the condition

$$
(\forall \eta \geq 0): \lambda_{\mathbf{b}}(\eta)<+\infty
$$

It is known that entire curve of bounded index can has every component of unbounded index. For a finite-dimensional curve such examples are contructed in [12]. Also it is known that if every component of entire curve $F: \mathbb{C} \rightarrow \mathbb{C}^{n}$ has bounded index then the curve is of bounded index (see [9]).

We prove first the following assertion.

Proposition 1. Let $l: \mathbb{C} \rightarrow \mathbb{R}_{+}$be a positive continuous function, each component $f_{j}$ of an entire curve $F: \mathbb{C} \rightarrow \ell^{\infty}$ be of bounded l-index, and $\sup \left\{N\left(f_{s} ; l\right): s \in \mathbb{N}\right\}$ be finite. Then $F$ has bounded l-index by the sup-norm with $N(F ; l) \leq \sup \left\{N\left(f_{s} ; l\right): s \in \mathbb{N}\right\}$.

Proof. In the proof we implicitly use the following elementary fact: "Let $A_{k, s}(z) \geq 0$ be continuous function in $\mathbb{C}, 0 \leq s \leq N, k \in \mathbb{N}$. Denote $A(z)=\sup \left\{A_{k, s}(z): 0 \leq s \leq N, k \in \mathbb{N}\right\}$, $A_{1}(z)=\sup _{0 \leq s \leq N} \sup _{k \in \mathbb{N}} A_{k, s}(z), A_{2}(z)=\sup _{k \in \mathbb{N}} \sup _{0 \leq s \leq N} A_{k, s}(z)$. Then $A_{1}(z)=A_{2}(z)=$ $A(z)$ for each $z \in \mathbb{C}$.'

In view of this fact for all $j \geq N=\sup \left\{N\left(f_{s}, l\right): s \in \mathbb{N}\right\}$ we have

$$
\begin{gathered}
\frac{\left\|F^{(j)}(z)\right\|_{\infty}}{j ! l^{j}(z)}=\frac{\sup \left\{\left|f_{s}^{(j)}(z)\right|: s \in \mathbb{N}\right\}}{j ! l^{j}(z)} \leq \\
\leq \sup \left\{\max \left\{\frac{\left|f_{s}^{(k)}(z)\right|}{k ! l^{k}(z)}: 0 \leq k \leq N\left(f_{s} ; l\right)\right\}: s \in \mathbb{N}\right\} \leq \\
\leq \sup \left\{\max \left\{\frac{\left|f_{s}^{(k)}(z)\right|}{k ! l^{k}(z)}: 0 \leq k \leq N\right\}: s \in \mathbb{N}\right\}= \\
=\sup \left\{\frac{\left|f_{s}^{(k)}(z)\right|}{k ! l^{k}(z)}: 0 \leq k \leq N, s \in \mathbb{N}\right\}=\max \left\{\frac{\left\|F^{(k)}(z)\right\|_{\infty}}{k ! l^{k}(z)}: 0 \leq k \leq N\right\},
\end{gathered}
$$

that is $N(F ; l) \leq \sup \left\{N\left(f_{s} ; l\right): s \in \mathbb{N}\right\}$. Proposition 1 is proved.

Positivity and continuity are weak restrictions on the function $l$. So, we will assume validity of some additional conditions which the function $l$ satisfies.

Let us denote

$$
\lambda_{\mathbf{b}}(\eta)=\sup _{t_{1}, t_{2} \in \mathbb{C}}\left\{\frac{l\left(t_{1}\right)}{l\left(t_{2}\right)}:\left|t_{1}-t_{2}\right| \leq \frac{\eta}{\min \left\{l\left(t_{1}\right), l\left(t_{2}\right)\right\}}\right\} .
$$

By $Q$ we denote the class of positive continuous function $l: \mathbb{C} \rightarrow \mathbb{R}_{+}$, satisfying the condition

$$
(\forall \eta \geq 0): \quad \lambda_{\mathbf{b}}(\eta)<+\infty
$$


Theorem 1. Let $l \in Q, F \in B^{\infty}$. If for each $\eta>0$ there exist $n_{0}=n_{0}(\eta) \in \mathbb{Z}_{+}$and $P_{1}=P_{1}(\eta) \geq 1$ such that for every $z_{0} \in \mathbb{C}$ there exists $k_{0}=k_{0}\left(z_{0}\right) \in \mathbb{Z}_{+}, 0 \leq k_{0} \leq n_{0}$, and

$$
\max \left\{\left\|F^{\left(k_{0}\right)}(z)\right\|_{\infty}:\left|z-z_{0}\right|=\frac{\eta}{l\left(z_{0}\right)}\right\} \leq P_{1}\left\|F^{\left(k_{0}\right)}\left(z_{0}\right)\right\|_{\infty} .
$$

then the function $F$ is of bounded l-index.

Proof. Our proof is based on the proof of an appropriate theorem for entire functions of one variable $[10,11,20,22]$. Suppose that for each $\eta>0$ there exist $n_{0}=n_{0}(\eta) \in \mathbb{Z}_{+}$and $P_{1}=P_{1}(\eta) \geq 1$ such that for every $z_{0} \in \mathbb{C}$ there exists $k_{0}=k_{0}(z) \in \mathbb{Z}_{+}, 0 \leq k_{0} \leq n_{0}$, for which inequality (4) holds. We choose $\eta>1$ and $j_{0} \in \mathbb{N}$ such that $P_{1} \leq \eta^{j_{0}}$. For given $z_{0} \in \mathbb{C}, k_{0}=k_{0}\left(z_{0}\right)$ and $j \geq j_{0}$ by Cauchy's formula for every component $f_{s}(z)$ as a function of one variable $z$ one has

$$
f_{s}^{\left(k_{0}+j\right)}\left(z_{0}\right)=\frac{j !}{2 \pi i} \int_{\left|z-z_{0}\right|=\eta / l\left(z_{0}\right)} \frac{f_{s}^{\left(k_{0}\right)}(z)}{\left(z-z_{0}\right)^{j+1}} d z \quad \forall s \in \mathbb{N} .
$$

Therefore, in view of (4) and (5) we have

$$
\begin{gathered}
\frac{\left\|F^{\left(k_{0}+j\right)}\left(z_{0}\right)\right\|_{\infty}}{j !}=\sup _{s \in \mathbb{N}} \frac{\left|f_{s}^{\left(k_{0}+j\right)}\left(z_{0}\right)\right|}{j !} \leq \sup _{s \in \mathbb{N}} \frac{1}{2 \pi} \int_{\left|z-z_{0}\right|=\eta / l\left(z_{0}\right)} \frac{\left|f_{s}^{\left(k_{0}\right)}(z)\right|}{\left|z-z_{0}\right| j+1}|d z| \leq \\
\leq \frac{1}{2 \pi} \frac{l^{j+1}\left(z_{0}\right)}{\eta^{j+1}} \int_{\left|z-z_{0}\right|=\eta / l\left(z_{0}\right)} \sup _{s \in \mathbb{N}}\left|f_{s}^{\left(k_{0}\right)}(z)\right||d z|=\frac{1}{2 \pi} \frac{l^{j+1}\left(z_{0}\right)}{\eta^{j+1}} \int_{\left|z-z_{0}\right|=\eta / l\left(z_{0}\right)}\left\|F^{\left(k_{0}\right)}(z)\right\|_{\infty}|d z| \leq \\
\leq \frac{l^{j}\left(z_{0}\right)}{\eta^{j}} \max \left\{\left\|F^{\left(k_{0}\right)}(z)\right\|_{\infty}:\left|z-z_{0}\right|=\frac{\eta}{l\left(z_{0}\right)}\right\} \leq P_{1} \frac{l^{j}\left(z_{0}\right)}{\eta^{j}}\left\|F^{\left(k_{0}\right)}\left(z_{0}\right)\right\|_{\infty},
\end{gathered}
$$

Obviously that $\frac{j ! k_{0} !}{\left(j+k_{0}\right) !} \leq 1$. Therefore, for all $j \geq j_{0}, z_{0} \in \mathbb{C}$

$$
\frac{\left\|F^{\left(k_{0}+j\right)}\left(z_{0}\right)\right\|_{\infty}}{\left(k_{0}+j\right) ! l^{k_{0}+j}\left(z_{0}\right)} \leq \frac{j ! k_{0} !}{\left(j+k_{0}\right) !} \frac{P_{1}}{\eta^{j}} \frac{\left\|F^{\left(k_{0}\right)}\left(z_{0}\right)\right\|_{\infty}}{k_{0} ! l^{k_{0}}\left(z_{0}\right)} \leq \eta^{j_{0}-j} \frac{\left\|F^{\left(k_{0}\right)}\left(z_{0}\right)\right\|_{\infty}}{k_{0} ! l^{k_{0}}\left(z_{0}\right)} \leq \frac{\left\|F\left(z_{0}\right)\right\|_{\infty}}{k_{0} ! l^{k_{0}}\left(z_{0}\right)}
$$

Since $k_{0} \leq n_{0}$, the numbers $n_{0}=n_{0}(\eta)$ and $j_{0}=j_{0}(\eta)$ are independent of $z_{0}$, this inequality means that the entire curve $F$ has bounded $l$-index and $N(F ; l) \leq n_{0}+j_{0}$. The proof of Theorem 1 is complete.

Theorem 1 presents one of the possible sufficient conditions of $l$-index boundedness. Proposition 1 and Theorem 1 lead to the following question:

Problem 3. Is it possible to deduce other known facts of theory of bounded index for space $B^{\infty}$, particularly, necessary conditions of l-index boundedness (for example see $[10,11,19$, 20])?

In this communication, we considered only the space $\ell_{\infty}$, because we did not know how to effectively introduce this concept in the case of other infinite-dimensional spaces, in particular in the space $\ell_{p}\left(p \in \mathbb{N}_{0}\right)$. 


\section{REFERENCES}

1. Baksa V.P., Analytic vector-functions in the unit ball having bounded $\mathbf{L}$-index in joint variables, Carpathian Mathematical Publications, 11 (2019), №2. (in print)

2. Baksa V.P., Bandura A.I., Skaskiv O.B., Analogs of Fricke's theorems for analytic vector-valued functions in the unit ball having bounded $\mathbf{L}$-index in joint variables, submitted to Proceedings of IAMM of NASU.

3. Bandura A., Skaskiv O., Sufficient conditions of boundedness of L-index and analog of Hayman's Theorem for analytic functions in a ball, Stud. Univ. Babeş-Bolyai Math., 63 (2018), №4, 483-501. doi:10.24193/subbmath.2018.4.06

4. Bandura A.I., Skaskiv O.B. Analytic functions in the unit ball of bounded L-index: asymptotic and local properties, Mat. Stud. 48 (2017), №1, 37-73. doi: 10.15330/ms.48.1.37-73

5. Bandura A.I., Some improvements of criteria of L-index boundedness in direction, Mat. Stud., 47 (2017), №1, 27-32. doi: 10.15330/ms.47.1.27-32

6. Bandura A., Skaskiv O., Asymptotic estimates of entire functions of bounded $\mathbf{L}$-index in joint variables, Novi Sad J. Math., 48 (2018), №1, 103-116. doi: 10.30755/NSJOM.06997

7. Bandura A., Petrechko N., Skaskiv O., Maximum modulus in a bidisc of analytic functions of bounded L-index and an analogue of Hayman's theorem, Mat. Bohemica, 143 (2018), №4, 339-354. doi: 10.21136/MB.2017.0110-16

8. Bandura A.I., Skaskiv O.B., Tsvigun V.L., Some characteristic properties of analytic functions in $\mathbb{D} \times \mathbb{C}$ of bounded L-index in joint variables, Bukovyn. Mat. Zh., 6 (2018), №1-2, 21-31.

9. Bordulyak M.T., Sheremeta M.M., Boundedness of l-index of analytic curves, Mat. Stud., 36 (2011), №2, 152-161.

10. Fricke G.H., Entire functions of locally slow growth, J. Anal. Math., 28 (1975), №1, 101-122.

11. Fricke G.H., Functions of bounded index and their logarithmic derivatives, Math. Ann., 206 (1973), 215-223.

12. Heath L.F., Vector-valued entire functions of bounded index satisfying a differential equation, Journal of Research of NBS, 83B (1978), №1, 75-79.

13. Kuzyk A.D., Sheremeta M.N., Entire functions of bounded l-distribution of values, Math. Notes, 39 (1986), №1, 3-8. doi:10.1007/BF01647624

14. Lepson B., Differential equations of infinite order, hyperdirichlet series and entire functions of bounded index, Proc. Sympos. Pure Math., 2 (1968), 298-307.

15. Nuray F., Patterson R.F., Multivalence of bivariate functions of bounded index, Le Matematiche, 70 (2015), №2, 225-233. doi: 10.4418/2015.70.2.14

16. Nuray F., Patterson R.F., Vector-valued bivariate entire functions of bounded index satisfying a system of differential equations, Mat. Stud., 49 (2018), №1, 67-74. doi: 10.15330/ms.49.1.67-74

17. Roy R., Shah S.M., Vector-valued entire functions satisfying a differential equation, J. Math. Anal. Appl., 116 (1986), №2, 349-362.

18. Roy R., Shah S.M., Growth properties of vector entire functions satisfying differential equations, Indian J. Math., 28 (1986), №1, 25-35.

19. Shah S.M., Entire function of bounded index. In: J.D. Buckholtz, T.J. Suffridge (eds.) Complex Analysis, Lecture Notes in Mathematics, V.599, 117-145, Springer, Berlin, Heidelberg, 1977.

20. Sheremeta M., Analytic functions of bounded index, Lviv: VNTL Publishers, 1999.

21. Sheremeta M., Boundedness of $l-M$-index of analytic curves, Visn. Lviv. Un-ty. Ser. Mech.-Math., 75 (2011), 226-231. (in Ukrainian)

22. Sheremeta M.N., Kuzyk A.D., Logarithmic derivative and zeros of an entire function of bounded l-index, Sib. Math. J., 33 (1992), №2, 304-312. doi:10.1007/BF00971102

Department of Advanced Mathematics

Ivano-Frankivsk National Technical University of Oil and Gas

Ivano-Frankivsk, Ukraine

andriykopanytsia@gmail.com 